\title{
A NEW METHODOLOGY FOR MEASURING THE FLOWABILITY OF GRANULAR MATERIALS
}

\author{
Ragunanth Venkatesh $^{1}$, Marko Bek ${ }^{1}$, Arkady Voloshin ${ }^{1,2}$, and Igor Emri ${ }^{1}$ \\ 1 University of Ljubljana, Faculty of Mechanical Engineering, Aškerčeva cesta 6, 1000, Ljubljana, \\ Slovenia. E-mail: ragunanth.venkatesh@fs.uni-lj.si, marko.bek@fs.ini-lj.si, igor.emri@fs.uni-lj.si \\ 2 Lehigh University, Mechanical Engineering, 19 Memorial Dr. West, Bethlehem, PA 18015, USA. E- \\ mail: avol4607@gmail.com
}

\section{Introduction}

Flow of granular material during processing, handling and transportation strongly influences the quality of the final product and its cost. Flowability (ability to flow) of granular materials depends on the characteristics of the material and on the conditions at which the flow is occurring. For example, the pharmaceuticals industries face complications on tablet filling where the flowability of granular materials plays a significant role. If the granular materials flow freely, it can ensure uniform filling of the feed from hoppers into the tableting equipment so that uniform mass and content of the tablet are maintained [1].

The previous paper [2] has proved the concept and working principle of GFA apparatus, a new methodology which was developed for predicting the flowability of powders and in paper [3], Schulze has found the interrelation between GFA index and Janssen's parameters (K-stress ratio and $\varphi$ internal friction angle between particle and wall). Janssen's parameters are standard method for evaluating the flowability of powders and are used for design of silos.

In this paper, the authors are presenting the upgraded GFA apparatus using optical measurement technique called digital image correlation to overcome the drawbacks related to usage of strain-gauges - Optical Granular Friction Analyzer (O-GFA). As an example, soil material was used to demonstrate the working principle of O-GFA.

\section{Concept and working of GFA}

The goal of the GFA is to obtain the pressure profile along the length of the cylinder filled with granular material and to introduce such profile as a measure of internal friction between particles of granular materials. The concept of the GFA is such that forces through the granular material and the pressure distribution along the length of the cylinder is a measure of how force is transmitted from the piston to the powder and from the powder to the cylinder. In this context, one expects that depending on the friction properties of the powder, the pressure will be transmitted in diversified ways along the cylinder, thus allowing us to obtain a method for determining internal friction between particles and condition at which the granular material will start to move.

Fig.1 illustrates how one can compare the internal friction properties of two granular materials using the GFA. Taking as a reference the pressure profile of a fluid which is constant along the length of the cylinder, one determines if certain powder has higher or lower internal friction by analyzing how steep is the pressure drop along the length of the cylinder, i.e. a granular material with less pressure drop will have lower internal friction (behavior closer to liquid) than another with very rapid pressure drop. Based on the literature, it is expected that powders with a smaller average particle size will exhibit higher internal friction [4,5]; for this reason, Powder 1 in Fig.1 has a much faster pressure drop than Powder 2.

Loading of the granular materials causes deformation to the cylinder, which is in the existing GFA apparatus measured using strain gauges at discrete locations in the axial and tangential direction as shown in Fig.1. From the measured strains, the axial pressure distribution is calculated. The internal friction of the granular material is represented by axial distribution of pressure within the measuring cylinder. As a measure of the internal friction Bek et al., defined a parameter called "GFA" index, defined as

$$
G F A_{I N D E X}=\frac{S_{\text {granular }}}{S_{\text {fluid }}}=\frac{\int_{0}^{L} p(z) d z}{p_{N F} \cdot L}=\frac{A}{F L} \int_{0}^{L} p(z) d z,
$$




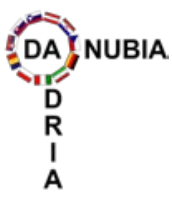

34th Danubia-Adria Symposium on Advances in Experimental Mechanics

University of Trieste, Italy, 2017 where $A$ is the area of the cylinder, $F$ is the force applied, $L$ is the length of the cylinder, $p(z)$ is the pressure obtained along the length of the cylinder for granular materials. The maximum value of the "GFA index" is 1 and indicates that tested granular material does not exhibit any internal friction, i.e., it behaves as a Newtonian fluid.

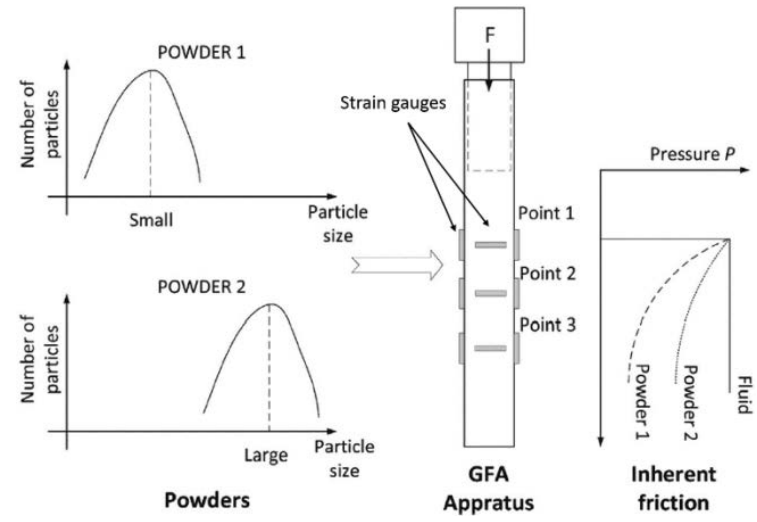

Fig. 1. GFA appartus (GFA) [2]

\section{Optical - GFA (O-GFA)}

Although the GFA apparatus proved the concepts in powder technology [3-5], it had certain drawbacks. It only had 3 measuring points as shown in Fig.1. By using optical measuring technique, one can increase the number of measurement points and consequently improve the accuracy of GFA index calculation according to Eq.1.

The GFA was upgraded using optical measurement setup commonly called as Digital Image Correlation (DIC). DIC is a non-contact optical experimental setup widely used in the field of experimental mechanics for measuring the surface deformation and characterization of the material. Through the 2D DIC setup one can measure the in-plane deformations (in $x$ and $y$ direction).

\subsection{Method of experiment}

Sand size $0-1 \mathrm{~mm}$ was used as testing sample. The sand was dried in oven for 5 hours to remove the humidity. Then the dried sand was filled into the entire cylinder length $(\mathrm{L}=295 \mathrm{~mm})$ and experiments were performed at room temperature. The applied load was 6764N (approx. pressure of 26MPa).

\subsection{Results}

The results show a sharp decrease of pressure profile along the length of the cylinder. The pressure drop is a strong indication that internal friction was large resulting in self-locking of sand particles.

The results, shown in Fig.2, clearly demonstrate that by using O-GFA one can obtain more number of measurement points, which improves the accuracy of GFA-index calculation.

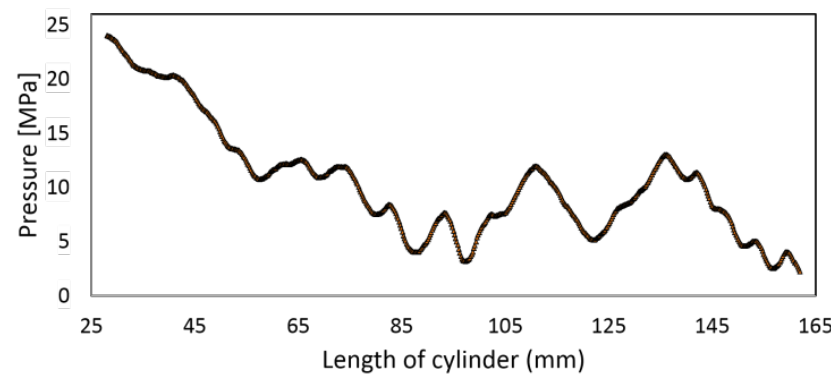

Fig. 2. Normal pressure profile calculated along the axial direction of the cylinder

\section{Summary and conclusions}

- A new upgraded granular friction analyzer (OGFA) was developed for evaluating the internal friction between granular particles.

- The number of measurement points were increased from 3 (GFA) to more than 100, which substantially improves the accuracy of GFA-index computation.

\section{Acknowledgements}

The authors would like to thank the financial support of the Slovenian Research Funding Agency (research program - P2-0264) and the young researcher grant (39190).

\section{References}

[1] Kato, Y., Ohkuma, M., Shimada, Y., Sunada, H., Evaluation of flowability of surface-modified preparations by the measurements of the interparticle adhesive force, J. Drug dev. Sci. Tech, (2005) 217-222.

[2] Bek, M., Gonzalez-Gutierrez, J.A., Moreno Lopez, Bregant, D., Emri, I., Apparatus for measuring friction inside granular materials - Granular friction analyzer, Powder Technol. 288 (2016) 255-265.

[3] Schulze, D., Comment on the paper “Apparatus for measuring friction inside granular materials granular friction analyzer”, Marko Bek et al., Powder Technol. 288 (2016) 255-265.

[4] Campbell, C.S., Granular material flows - An overview, Powder Technol. 162 (2006) 208-229.

[5] Rao, K.K., Nott, P.R., An Introduction to Granular Flow, Cambridge University Press, New York, 2008. 